\title{
A Newly Dielectric Embedded Quasi-Microstrip Yagi Antenna with Barrier
}

\author{
Guoqi $\mathrm{Ni}^{1,2}$ Benqing Gao ${ }^{1}$ Junwei $\mathrm{Lu}^{3}$ \\ 1. Beijing Institute of Technology, 100081 China \\ 2. Guilin air Force Academy, 100081China \\ 3. Griffith University, 4111 Australia
}

\begin{abstract}
This paper presents a newly Dielectric Embedded Quasi-Microstrip Yagi Antenna with Barrier (DE-QMYAB). It is a improved structure from Dielectric Embedded Quasi-Microstrip Yagi Antenna (DE-QMYA).The parasitic patches are arranged nearby each main element patch. So as to the configuration of reflectors are formed. The electromagnetic wave is bared by the parasitic patches. This results in antenna performance improvement, including gain and bandwidth.
\end{abstract}

\section{INTRODUCTION}

The microstrip Yagi antenna is a plane type. It is researched by application of Yagi principle in microstrip antenna. There are many advantages for Yagi antenna and microstrip antenna $a^{[1 \sim 5]}$. Therefore, they have been widely applied. Their many element patches are printed on a plane of microstrip substrate, and their half of surface is denuded in air. The beam peak of this antenna isn't in vertical direction with plane of microstrip substrate. It is not convenient that the antenna is use to conformalness with other object. In this paper, a newly DE-QMYAB is researched by Yagi principle and dielectric embedded principle. This antenna's configuration is consisted of microstrip element patches spliced along radiation direction that it is the same as in the air, i.e., all microstrip element patches are not printed on a plane of microstrip substrate. The each microstrip element patch is printed on a plane of different microstrip substrate. These microstrip element patches are devolution spliced along radiation direction by microstrip substrates (means: one by one). It is more like a sandwich configuration. The each microstrip element patch is embedded in those microstrip substrates. It is a three-dimensional configuration relative to the microstrip Yagi antenna. But this antenna performance is affected due to thickness of microstrip substrates. This paper presents an improved antenna scheme, namely, that is DE-QMYAB. but its performance is improved markedly.

\section{The Structure ImProved And Result of DE-QMYA}

For our first structure (DE-QMYA), its gain is decreased markedly, because it is complicated that electromagnetic wave propagates in dielectric. We believe it is due to the effect of director element that do not play the itself role in it Therefore, in accordance with microstrip antenna theory ${ }^{[6]}$, the parasitic patches are mounted on both sides of main element patches(except reflection element patch), their width is same as the main element patches, but slightly longer than the length of their main element patches. These parasitic patches are called as
"Barrier". So as to the electromagnetic wave propagates along the antenna radiation direction. Thereby, the directional performance of antenna is improved.

The actual structure of DE-QMYAB is designed, shown in Figure 1 (note: the figure shows that it only is patches, but the dielectric layers are neglected). In the figure, the reflection element is a rectangular patch. The driven elements are symmetric patches, which stands in the central position of the figure in the $\mathrm{z}$ axis. The patches on both sides of the driven element patches are parasitic element patches. It is called as the "barrier". The patch in the front of the driven element patches is a director element A (see fig. 1). There are two parasitic patches on both sides of the director element A. The patch in the front of the director element $\mathrm{A}$ is a director element $\mathrm{B}$. There are two parasitic patches on both sides of the director element B. It filled with dielectric among element patches.

The simulated result of the antenna improved is very satisfied. Based on its size, a physical antenna mode is manufactured and its performance has been measured. The all results are shown in Table 1and 2. In Table 1and 2, the data of the pre-improved (without the "barrier") mode is also shown (that is, without the "barrier"). Compared DE-QMYA to DE-QMYAB, the beam breadth of radiation pattern is varied from $71^{\circ}$ to $59^{\circ}$, and the bandwidth is varied from $0.14 \mathrm{GHz}$ to $0.261 \mathrm{GHz}$, and antenna's Gain is varied from 6.4575 to $9.7371 \mathrm{~dB}$, and the real input impedance from $57 \Omega$ to $54 \Omega$. The $\mathrm{S} 11$ curves of the modes are shown in Figure 2, we can see that working bandwidth increased significantly.

For the results of two modes, the agreement between simulation and measurement is good (see table land 2). It shows that our scheme is correct, these results may provide a useful reference for research DE-QMYA in future.

\section{The ANALYSIS OF EXPERIMENTAL RESUltS}

Due to comparison of the results from the above, we can see that function of the "barrier" appended on both sides of element patches is obvious. The antenna performance have been improved significantly. Below we make an analysis.

\section{A. On Analysis of the Antenna Performance Improvement}

After the "barrier" is appended, the spacing between the main elements and the "barriers" is appropriately adjusted, so that the electromagnetic waves are Superposition in phase between the reflection electromagnetic waves of the "barrier", 


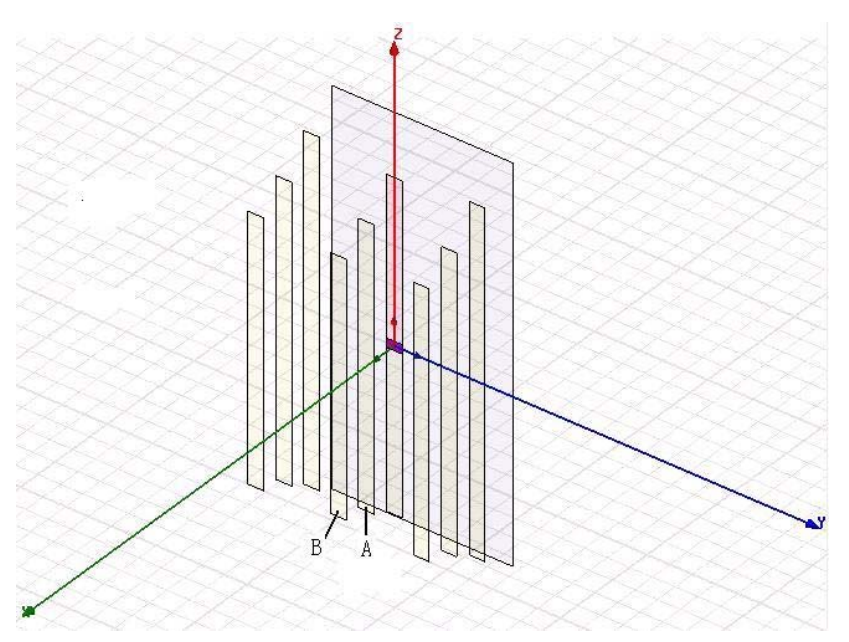

Figure 1. Configuration of DE-QMYAB

and radiation the electromagnetic waves of main element. The antenna performance is improved. As we design, it is to let the length of each "barrier" longer than that of the main element. So, it is more reasonable to explain the phenomenon with Yagi antenna reflector element theory ${ }^{[6]}$. There, it is "barrier" that is the reflection element. The electromagnetic wave in the places, the left side, right side and rear have been "stopped", was forced to lead the front direction. Therefore, it make better for the directional performance of antenna.

The other contributors of improve directional performance of antenna is the dielectric. According to theory of electromagnetic fields ${ }^{[7]}$, the electromagnetic wave from the dense medium to the sparse medium takes place complete reflection, where an angle of incidence is greater than the critical angle in both the medium interface. The reference also provides for an example of the principle used this antenna in the case. That it is dual reflector antenna with dielectric guide. The reference also provides for the principle of this antenna in the case. That it is dielectric guide dual reflector antenna. In this antenna, the sparse medium is air, and the dense medium is the dielectric

TABLE I

\begin{tabular}{|c|c|c|c|c|c|}
\hline \multicolumn{6}{|c|}{ PARAMETER VALUE OF DE-QMYAB AND DE-QMYA } \\
\hline Mode & Way & fo(GHz) & $\mathrm{G}(\mathrm{dB})$ & $\mathrm{B}(\mathrm{GHz})$ & $\rho$ \\
\hline \multirow[t]{2}{*}{ DE-QMYA } & Simulation & 2.40 & 6.4575 & 0.14 & 1.15 \\
\hline & measurement & 2.407 & 6.37 & 0.142 & 1.2 \\
\hline \multirow[t]{2}{*}{ DE-QMYAB } & Simulation & 2.401 & 9.7371 & 0.261 & 1.15 \\
\hline & measurement & 2.399 & 9.8011 & 0.260 & 1.18 \\
\hline
\end{tabular}

TABLE II

PARAMETER VALUE OF DE-QMYAB AND DE-QMYA

\begin{tabular}{|l|l|l|l|l|}
\hline Mode & Way & $\theta_{0.5 \mathrm{E}}\left({ }^{\circ}\right)$ & $\operatorname{Zre}(\Omega)$ & $\operatorname{Zim}(\Omega)$ \\
\hline \multirow{2}{*}{ DE-QMYA } & Simulation & 71 & 57 & -2 \\
\cline { 2 - 5 } & measurement & 72 & 56 & \\
\hline \multirow{2}{*}{ DE-QMYAB } & Simulation & 59 & 54 & -1 \\
\cline { 2 - 5 } & measurement & 61 & 52 & \\
\hline
\end{tabular}

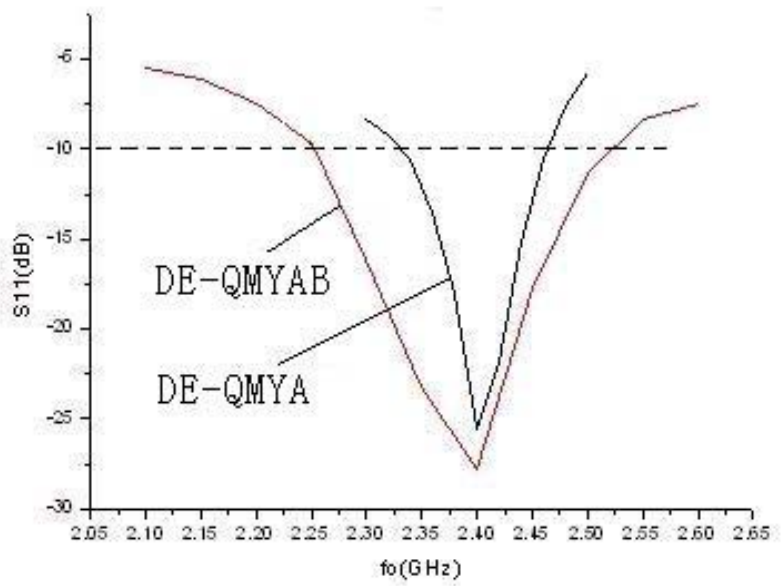

Figure 2. The $\mathrm{S} 11$ curve of DE-QMYAB and DE-QMYA

( $\left.\varepsilon_{r}>1\right)$. The spacing between the auxiliary plane and radiator of antenna is decreased by the antenna structure, and antenna size is decreased. The reference [8] provides another example of directional antenna enhanced by dielectric. Namely it is dielectric haulm antenna.

\section{B. Analysis on the Improvement of the Bandwidth of the Antenna}

According to microstrip antenna theory, the microstrip antenna resonant is changed by the increased parasitic patch, so that the single-tuning characteristics of the microstrip antenna is changed into a dual-tuner, the resonant peak curve becomes broad and flat, thus variable bandwidth becomes wide ${ }^{[9]}$.

According to microstrip antenna cavum model analysis method, the microstrip antenna is a resonant antenna, so that its bandwidth is narrow, and its resonant characteristics depend on a high-Q parallel resonant circuit. Therefore, the basic way of extending bandwidth is that the $Q$ value of equivalent resonant circuit is decreased ${ }^{[10]}$, such as increasing the substrate thickness $h$, and reducing the relative dielectric constant of the substrate, and increases length / width ratio of rectangular patch, and so on.. In the antenna, the thickness of the substrate is increased, but the relative dielectric constant $\mathcal{E}_{r}$ is also greater, thus it is the little that the performance of antenna is improved. So, the method of amending resonant circuit must be adopted. The simple RLC equivalent circuit is adapted to coupling resonant circuit with the multi-resonant points. The parasitic patches are printed conformally nearby the side of the main element patch, this is precisely to form the method of multiantenna resonant point. Therefore, the bandwidth of the antenna is increased to the $0.261 \mathrm{MHz}$. The relative ratio of bandwidth:

$$
B W_{c e}=\frac{B}{f_{0}}=\frac{0.261}{2.40} \approx 11 \%
$$

Note: the value of formula (1) is a measurement value of the improved mode with the parasitic patch antenna. 
However, the $Q$ value without the parasitic patch antenna can be calculated, when cavum model analysis method is used.

Namely, Q=17.43.

So, the relative ratio of bandwidth of no printed the parasitic patch antenna can be calculated, when $\rho \leq 2$,

$$
B W=\frac{\rho-1}{\sqrt{\rho} Q} \times 100 \%=4.1 \%
$$

The relative ratio of bandwidth without printed the parasitic patch antenna have been measured:

$$
B W_{e}=\frac{B_{c}}{f_{0}}=\frac{0.14}{2.40} \approx 5.8 \%
$$

From above value (2) and (3): for the first antenna (without "barrier") theoretical value and the actual measured value is closer. From above value (1) and (3): the actual measured values of the mode with "barrier", is twice to that of the first mode.

\section{Conclusions}

From above results of the analysis, we can see that gain and bandwidth of the antenna with the "barrier" is the most obviously improved. Results of the simulation are in good agreement with the physical antenna measured. It is showed that our newly antenna scheme is nice scheme. In the future, we will do for other research to improve DE-QMYA performance.

ACKNOWLEDgMent
During Australia the research, I would like to thank Min Shi, and Xiao Duan, and Zhaohui Sun, and other people for me help.

\section{REFERENCES}

[1] John Huang, Arthur C,"Densmore. Microstrip Yagi Array Antenna for Mobile Satellite Vehicle Application ", IEEE transaction on antennas and propagation, Vol.39, NO.7, July 1991, pp.1024-1030.

[2] Naoki Honma, Tomohiro Seki, etc, "Compact Six-Sector Antenna Employing Three Intersecting Dual-Beam Microstrip Yagi-Uda Arrays With Common Director", IEEE transaction on antennas and propagation, Vol.54, NO.11, November 2006, pp.3055-3062.

[3] Derek Gray, Jun Wei Lu, and David V.Thiel, "Electronically Steerable Yagi-Uda Microstrip Patch Antenna Array", IEEE transaction on antennas and propagation, Vol.46, NO.5, May 1998, pp. 605-608.

[4] Y. Qian, W.R. Deal, N. Kaneda and T. Itoh, " Microstri p-fed quasi-Yag i antenna with broad band characteristics", ELECTRONICS LETTERS 12th, November 1998,Vol. 34 No. 23, pp.2194-2196.

[5] Jun Guo, Feng Wang, Mouping Jin, "Design of a Microstrip-Fed Quasi-Yagi Antenna with Wide Bandwidth. Modern Electronics", No.2, February. 2002, pp.25- 27.

[6] Xuyi Li, "Electromagnetic Field and MicroWave Technology (Second Edition)", South China University of Technology Press, Guangzhou of China,Sepember. 2004, pp.264-271.

[7] Xingiian Kang, “Antenna Principle and Design”, National Defence Industry Press, Beijing of China, November. 1995, pp.261-262.

[8] John D.Kraus, Ronald J.Marhefka, “Antennas: For All Applications (Third Edition)", Publishing House of Electronics Industry, Biejing of China, July. 2005, pp.157-159.

[9] Wanzheng Lu, “ Antenna Theory and Technology”, Xidian University Press. Xian of China, May, 2004, pp.245-247.

[10] Shunshi Zhong, "Microstrip Antenna Theory and Application", Xidian University Press. Xian of China, June.1991, pp.152-156. 